\title{
MAINTENANCE OF \\ CONSTANT WAVE PARAMETERS BY SPERM FLAGELLA AT REDUCED FREQUENCIES OF BEAT
}

\author{
By C. J. BROKAW AND R. JOSSLIN \\ Division of Biology, California Institute of Technology, Pasadena, \\ California 9 I 109
}

(Received 24 April 1973)

INTRODUCTION

The bending of flagella and cilia appears to be generated by a sliding filament process resembling the sliding filament mechanism for muscle contraction (Satir, I968; Summers \& Gibbons, I97I). If the active sliding process in flagella is locally regulated by the curvature of the flagellum, a feedback loop is established which can cause the spontaneous initiation and propagation of bending waves along the flagellum (Brokaw, 1971, 1972a). Further development of this mechanism for the propagation of the flagellar bending wave leads to a search for the factors responsible for controlling the parameters of the propagated bending waves. The wavelength of the bending waves could be controlled by a factor equivalent to an internal viscous bending resistance (Brokaw, I972a, $c$ ). Changes in wavelength observed when sperm flagella are moving in media of increased viscosity are consistent with this hypothesis (Brokaw, 1972d). According to this hypothesis the determination of the wavelength of the propagated bending waves is independent of the properties of the active sliding process, and inhibitors of the active sliding process should not alter the wavelength.

In the first quantitative study of the movements of the sea-urchin sperm flagellum, James Gray noted that in some spermatozoa approaching the end of their active life, the frequency of the flagellar beat decreased gradually without any drastic change in the form of the waves (Gray, 1955). Gray's Fig. 3 shows a sequence of tracings from a cine-film record of the movement of such a spermatozoon, which was swimming with a frequency of $0.55 \mathrm{~Hz}$ and a waveform which was nearly normal, but which had somewhat larger than normal values of wavelength and amplitude. Some similar observations have been reported in more recent work but have not been examined in detail.

In the present study the behaviour of the wavelength and amplitude of flagellar bending waves at reduced frequencies has been studied in greater detail using three different experimental situations to obtain reduced frequencies. Our results confirm Gray's original observation: substantial changes in frequency can occur with no detectable change in wavelength or wave amplitude, although at very low frequencies increases in wavelength and amplitude appear. The results agree with the hypothesis that wavelength is regulated by a factor equivalent to an internal viscous bending resistance, but no satisfactory explanation for the regulation of amplitude is apparent. 


\section{CIONA SPERMATOZOA INHIBITED WITH TRITON X-IOO}

\section{Materials and Methods}

Spermatozoa were collected from the tunicate, Ciona intestinalis, by dissection (Brokaw \& Benedict, $1968 b$ ) and diluted with filtered sea water containing $2 \mathrm{mM}$ histidine, $0.5 \mathrm{mM}$ EDTA, and $2 \%$ polyvinylpyrollidinone (PVP) at $\mathrm{pH} 8 \cdot 1$. All observations were carried out in a room maintained at $16{ }^{\circ} \mathrm{C}$. Spermatozoa swimming at the upper surface of an open drop on a microscope slide were photographed on Kodak Tri-X film using a Zeiss $25 \mathrm{X}$ planapochromat objective and a Chadwick-Helmuth model 72 flash lamp and model ${ }_{13} 6$ power supply. Multiple flash photographs were taken at known flash frequencies using dark-field illumination, and wave parameters were determined on the photographic prints.

Reduced frequencies were obtained by adding small amounts (up to $0.01 \%$ ) of the non-ionic detergent, Triton X-IOO, to the sperm suspension. Larger concentrations of this detergent stop the movement completely and remove the membranes from sperm flagella (Gibbons \& Gibbons, I972). The low concentrations of Triton we used produced frequencies considerably less than normal, which remained nearly constant for 5-10 min. Although the exact effect of Triton at low concentrations is unknown, it could cause a reduction in adenosine triphosphate (ATP) concentration within the flagellum to a lower than normal steady-state level, either by increasing the leakage of ATP through the flagellar membrane, or by decreasing the rate of supply of ATP by metabolism.

\section{Results}

Plate I, fig. I, illustrates a typical result for a Ciona spermatozoon swimming in the absence of Triton. This spermatozoon executed 5.84 cycles of beating in 6 flash intervals, so its frequency is $38.6 \mathrm{~Hz}$, just slightly less than the flash frequency of $39.7 \mathrm{~Hz}$. Lines were inscribed on enlarged prints through the 'straight regions' which appear to separate the regions of bending in each wave pattern. Points on these lines were selected as the inflexion points between bends, indicating the ends of one complete bending wave. The wavelength, $L$, measured along the flagellum, was determined by measuring the distance along the flagellum between inflexion points with a piece of flexible polyethylene tubing. The angles between the lines through the straight regions were measured to determine the total angle of bend in each of the two bent regions of this wave. Most bending waves are somewhat asymmetrical, so that the angles of the two bent regions of a wave are unequal. Gibbons \& Gibbons (1972) suggested that the larger angle be designated the 'principal bend' and the smaller angle the 'reverse bend'. We found no consistent changes in the relative values of principal and reverse bends in these experiments and have therefore presented only the average bend angle for each spermatozoon.

I 48 photographs of movement of Cionc: spermatozoa with and without Triton were measured as described in the preceding paragraph. These measurements are summarized in Text-fig. I. The frequencies ranged from $40 \mathrm{~Hz}$ down to ${ }_{1} \cdot 5 \mathrm{~Hz}$, which was the lowest stable frequency we could obtain with Triton-inhibition. Some typical examples of the movement of Ciona spermatozoa at low frequencies are shown in Plate I, figs. 3, 4 . 


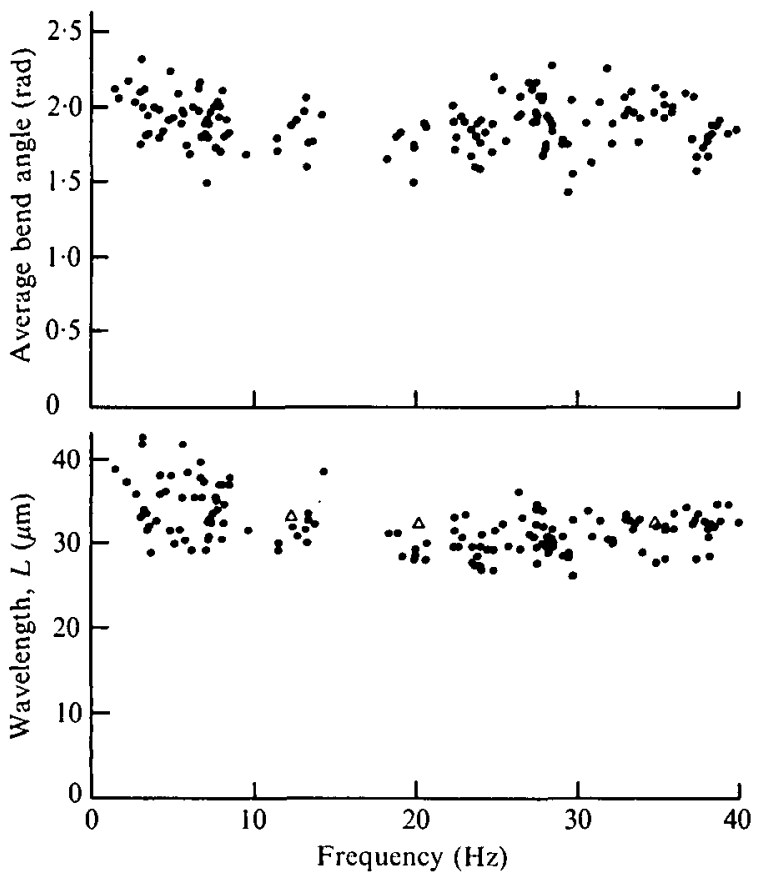

Text-fig. I. Wave parameters for 148 Ciona spermatozoa treated with varying amounts of Triton X-Ioo. The three points indicated by open triangles are from another study (Brokaw \& Benedict, $1968 b$ ) in which Ciona spermatozoa were measured in solutions containing $0.0,0.12$, or $0.18 \mathrm{M}$ thiourea.

The results in Text-fig. I indicate that over the fourfold range of frequencies from IO- $40 \mathrm{~Hz}$ the wavelength of the flagellar bending waves remains essentially constant in the range of $28-34 \mu \mathrm{m}$. Below $10 \mathrm{~Hz}$ the wavelength increases slightly. The 25 spermatozoa with the highest frequencies, ranging from 33.6 to $40.0 \mathrm{~Hz}$, had a mean wavelength of $32 \mu \mathrm{m}$. The 25 spermatozoa with the lowest frequencies had frequencies ranging from $\mathrm{I} \cdot 5$ to $6.2 \mathrm{~Hz}$ and a mean wavelength of $36.5 \mu \mathrm{m}$. This $14 \%$ increase in wavelength in the low-frequency group is statistically significant at well beyond the $99 \%$ level.

The measurements of bend angle fail to show any statistically significant change over the range of frequencies we studied. Although there is considerable scatter in the measurements, a $10 \%$ change in bend angle between the low-frequency and highfrequency groups would be statistically detectable. Therefore the bend angle of the bending waves of Ciona sperm flagella remains constant within $\pm 10 \%$ as the frequency is varied over the range of $\mathrm{I} \cdot 5$ to $40 \cdot 0 \mathrm{~Hz}$ by treatment with Triton X-100.

Text-fig. I also contains three data points from an earlier study (Brokaw \& Benedict, $1968 b$ ) in which frequency and wavelength were measured for samples of Ciona spermatozoa in normal sea water and in the presence of 0.12 or $0.18 \mathrm{~m}$ thiourea. The thiourea-inhibited spermatozoa also show no significant change in wavelength over the range of $12-35 \mathrm{~Hz}$.

In addition to the data in Text-fig. I, we also obtained 6 photographs of spermatozoa beating at approximately $3 \mathrm{~Hz}$ which had much less regular wave patterns, as illustrated by Plate I, figs .5, 6. In these cases the bending wave usually occupied more than 
the length of the flagellum so that a wavelength could not be determined by the method used for the other photographs. By measuring the position of inflexion points at successive flash intervals to obtain estimates of propagation velocity, we determined that the apparent wavelength of the movement in these cases was in the range of 60-90 $\mu \mathrm{m}$, much greater than the values shown in Text-fig. I. However, in these spermatozoa 'wavelength' is not well defined because the bends change significantly in size and shape as they are propagated along the flagellum. A bend angle for these spermatozoa was also difficult to estimate because the bend angles changed significantly as the bends were propagated, and because these spermatozoa showed larger differences between the principal and reverse bends. However, there does not appear to be any major or consistent difference between the bend angles in these 6 spermatozoa and in the larger sample.

\section{LYTECHINUS SPERMATOZOA REACTIVATED WITH VARIOUS ATP CONCENTRATIONS}

\section{Materials and Methods}

Glycerinated spermatozoa from the sea urchin, Lytechinus pictus, were prepared as described by Brokaw $(1966,1967)$ and used within a few hours after preparation. The reactivation solution was similar to that used by Brokaw \& Benedict (1968a) and contained $0.25 \mathrm{M} \mathrm{KCl}$, $0.10 \mathrm{M}$ urea, $4 \mathrm{mM} \mathrm{MgSO}_{4}, 5 \mathrm{~mm}$ thioglycollate, $2 \% \mathrm{PVP}$, $0.3 \%$ bovine serum albumin, and $0.02 \mathrm{M}$ HEPES buffer $(N$-2-hydroxyethylpiperazine- $N^{\prime}$-2-ethanesulfonic acid) at $\mathrm{pH} 7.8$. In addition to various concentrations of ATP we added $0.05 \mathrm{~mm}$ adenosine diphosphate (ADP) to minimize changes in conditions during the experiment resulting from accumulation of ADP from dephosphorylation of ATP. Approximately $2 \mu \mathrm{l}$ of glycerinated sperm suspension were added to $200 \mu \mathrm{l}$ of re activation solution in a glass well slide and mixed very gradually. A drop of this mixture was then transferred to a slide on the microscope stage and covered with a cover glass. The room temperature was maintained at $16^{\circ} \mathrm{C}$ and, in addition, some of the frequency measurements were made on a microscope with a temperaturecontrolled stage at $16^{\circ} \mathrm{C}$. Multiple-exposure darkfield flash photomicrographs of individually selected, swimming spermatozoa were made on Kodak 2485 high-speed recording film using a Zeiss $40 X$ oil immersion apochromat objective. A stroboscopic measurement of the frequency of each spermatozoon photographed was also recorded.

Although very gentle handling of small quantities of glycerinated spermatozoa gave somewhat higher percentages of motile spermatozoa than in some earlier experiments (Brokaw \& Benedict, $1968 a$ ), only a small percentage of spermatozoa in the suspensions were judged to be suitable for photographic measurement. Most of the spermatozoa observed were either non-motile, or wholly or partially stuck to the slide or cover glass, or abnormally motile due to visible breakage or swelling of the sperm flagellum. Of the remaining fraction of freely swimming motile spermatozoa, some were eliminated if their movements were not consistent through the period of a few seconds required for selection, frequency measurement, positioning and focusing, or if the tail did not appear to be uniformly refractile along its length. Finally, there was a less critical tendency to exclude apparently abnormal spermatozoa whose bending waves did not pass along the full length of the flagellum and a tendency to favour spermatozoa with. 


\section{Table I. Parameters of the movement of glycerinated sea-urchin spermatozoa}

$\begin{array}{lcccc}\text { ATP concentration (mM): } & 3 \cdot 0 & 1 \cdot 0 & 0.33 & 0.1 \mathrm{I} \\ \text { Number of spermatozoa measured: } & 3 \mathrm{I} & 33 & 22 & 27\end{array}$

Means and estimated standard deviations of measurements on individual spermatozoa:

\begin{tabular}{|c|c|c|c|c|}
\hline$f=$ beat frequency $\left(\sec ^{-1}\right):$ & $24 \cdot 5 \pm 2 \cdot 3$ & $18 \cdot 4 \pm I \cdot 6$ & $10 \cdot 7 \pm 1 \cdot 3$ & $4 \cdot 8 \pm 0.7$ \\
\hline $\begin{array}{c}L=\text { wavelength measured along } \\
\text { the flagellum }(\mu \mathrm{m}):\end{array}$ & $25 \cdot 4 \pm r \cdot 6$ & $26 \cdot 8 \pm I \cdot 8$ & $28 \cdot 2 \pm I \cdot 5$ & $32 \cdot 9 \pm 2 \cdot 7$ \\
\hline $2 \theta_{0}=$ angle of principal bend (rad): & $2.30 \pm 0.22$ & $2.51 \pm 0.19$ & $2 \cdot 76 \pm 0 \cdot 22$ & $3.02 \pm 0.20$ \\
\hline $2 \theta_{0}^{\prime}=$ angle of reverse bend $(\mathrm{rad}):$ & $0.42 \pm 0.28$ & $0.66 \pm 0.29$ & $0.89 \pm 0.26$ & $1 \cdot 20 \pm 0.33$ \\
\hline$\rho=$ radius of principal bend $(\mu \mathrm{m}):$ & $4 \cdot 6$ & $4 \cdot 5$ & $4 \cdot 6$ & $5 \cdot I$ \\
\hline$\rho^{\prime}=$ radius of reverse bend $(\mu \mathrm{m}):$ & 16 & 13 & 12 & II \\
\hline
\end{tabular}

more symmetrical wave patterns. Two or three exposures were usually made of each spermatozoon and the best one, or occasionally two, photographs were selected for printing and measurement. Additional selection was made at this stage because some spermatozoa were eliminated if no properly focused photographs were obtained.

\section{Results}

Photographs of motile, ATP-reactivated spermatozoa were obtained at four ATP concentrations $(3 \cdot 0,1 \cdot 0,0.33$, and $0.1 \mathrm{I} \mathrm{mM})$. Selected examples of these photographs are shown are shown in Plate I, figs. 7-10. Wavelengths and bend angles were measured on the photographs using methods similar to those used with Ciona spermatozoa. The results of these measurements are summarized in Table $\mathrm{I}$.

Additional stroboscopic measurements of frequency were made with these preparations at various ATP concentrations in order to obtain a more complete sampling of the relationship between frequency and ATP concentration. These additional measurements of frequency and the frequencies of the photographed sperm samples are compared in Text-fig. 2. This comparison demonstrates that, at least with respect to frequency, the photographed spermatozoa are representative of a somewhat larger population of motile spermatozoa. Frequencies of reactivated spermatozoa are related to ATP concentration by typical Michaelis-Menten kinetics (Brokaw, I967; Holwill, 1969). A double reciprocal (Lineweaver-Bürk) plot of the data shown in Text-fig. 2 yields values of $29.4 \mathrm{~Hz}$ for $f_{\max }$ and $0.55 \mathrm{mM}$ for $K_{n}$ (equal to the ATP concentration at which $f=\frac{1}{2} f_{\max }$ ). These values differ somewhat from values reported earlier of $25.6 \mathrm{~Hz}$ and $0.43 \mathrm{mM}$, respectively (Brokaw, 1967 ). The difference may be a reflexion of the lower thioglycollate concentration and higher viscosity of the reactivation solution used in the earlier study.

The measurements show a small increase in wavelength, $L$, as the frequency decreases with decreasing ATP concentration. These differences between mean values of wavelength at different ATP concentrations are statistically significant at better than the $99 \%$ level. Both the principal and reverse bend angles increase with decreasing frequency. Their ratio does not remain constant; instead, the absolute difference between them is constant. 


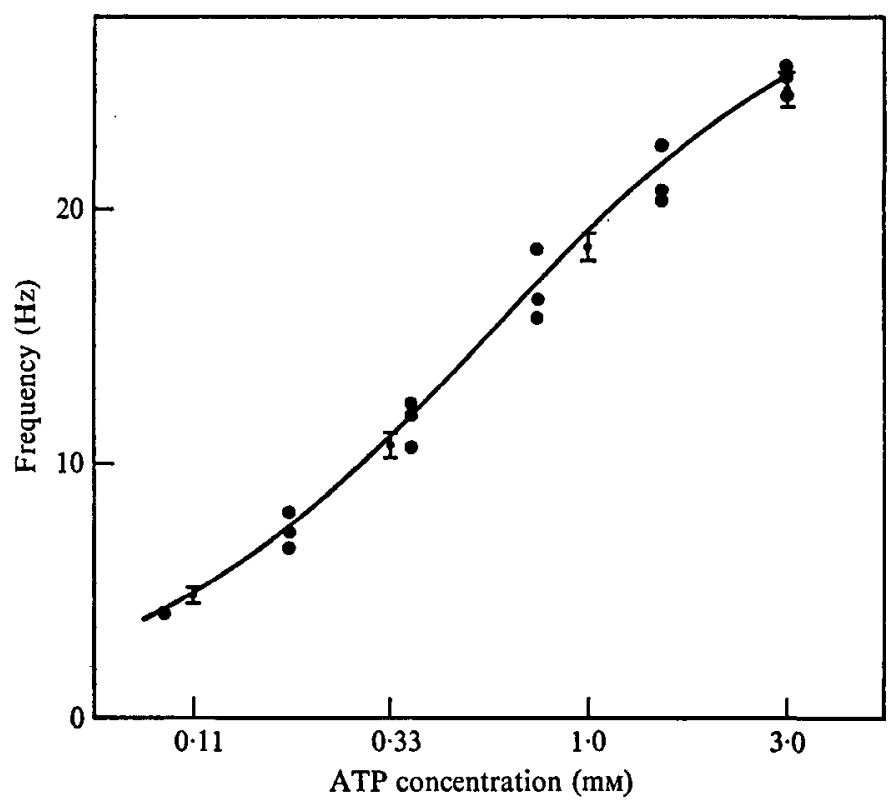

Text-fig. 2. Frequencies of Lytechinus spermatozoa reactivated at various ATP concentrations. The points with standard deviations indicated are for the measurements on sperm photographs. Each of the other points represents the mean frequency of a sample of 20 spermatozoa, measured stroboscopically. The curve is obtained from the straight line which best fits these points on a double reciprocal (Lineweaver-Bürk) plot.

\section{DECAPITATED LYTECHINUS SPERMATOZOA}

\section{Materials and Methods}

Spermatozoa from the sea urchin, Lytechinus pictus, were suspended in a filtered sea-water solution containing $0.5 \mathrm{mmEDTA}, 1 \%$ PVP, 0.0 M histidine, 0. I M thiourea, and $0.2 \%$ methyl cellulose at $\mathrm{pH} 8 . \mathrm{r}$. The suspension was drawn in and out of a fine glass pipette several times, quickly transferred to a microscope slide and covered with a cover glass. In this way it was possible to obtain a small number of decapitated spermatozoa which were swimming at about half the normal frequency (Brokaw, 1965, 1970). Decapitated spermatozoa were photographed automatically at 2 sec intervals using a Zeiss $40 \mathrm{X}$ apochromat objective and Kodak Tri-X film. The flash frequency for each photograph was recorded by a counter and printer. By this means a photographic record was obtained of the movement of an individual flagellum as its movement slowed down, presumably because of exhaustion of its ATP supply.

These photographs were measured in a manner similar to that used by Brokaw (1970). Each image was fitted with circular arcs and straight lines to obtain the location of the ends of bent regions relative to the head end of the flagellum and the angles of each bend. The measurements were plotted as in Text-figs. I and 2 of Brokaw (1970) and the frequency, mean propagation velocity, and maximum bend angle were obtained from these plots.

\section{Results}

Plate I, figs. II-20, show the photographs obtained from the experiment which yielded the most complete results. This sequence covers an 18 sec period during which 


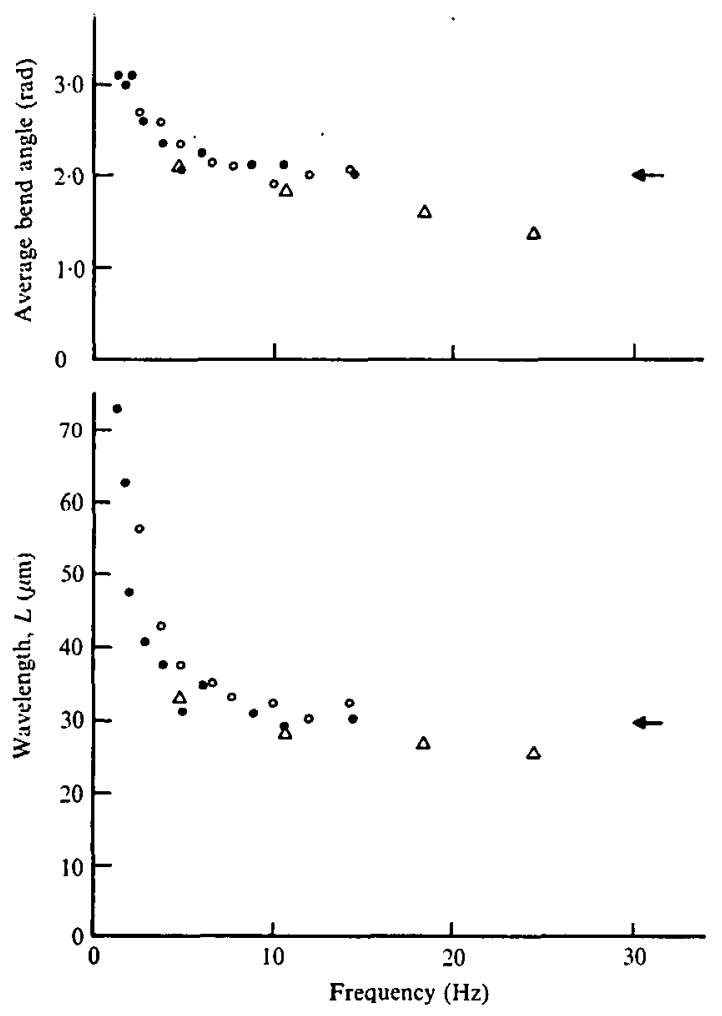

Text-fig. 3. Wave parameters for Lytechinus spermatozoa. The solid circles are obtained from measurements on the photographs of a decapitated sperm flagellum shown in Plate $I$, figs. I I-2O. The open circles were obtained from measurements on a series of photographs of another decapitated sperm flagellum. The open triangles are data from Table 1 , for the ATP-reactivated sperm samples. Values for the bend angle and wavelength of normal Lytechinus spermatozoa (Brokaw, 1965) are indicated by arrows.

the frequency decreased from $\mathrm{I}_{4} \cdot 5 \mathrm{~Hz}$ to $\mathrm{I} \cdot 3 \mathrm{~Hz}$. The waveform in the first three photographs (frequency greater than $8 \mathrm{~Hz}$ ) is similar to that of spermatozoa at their normal frequency of approximately $3 \circ \mathrm{Hz}$ (Brokaw, 1965), but at lower frequencies the size and shape of the bending waves changes. Text-fig. 3 shows measurements of the wavelength and the average value of the principal and reverse bend angles for this and one other decapitated spermatozoon. Both the bend angles and the wavelengths show very little change as the frequency decreases from $30 \mathrm{~Hz}$ down to $8-10 \mathrm{~Hz}$. Below $8 \mathrm{~Hz}$ both the bend angles and wavelengths measured on the decapitated spermatozoa become much larger than normal, with a sharp increase at frequencies below about $3 \mathrm{~Hz}$.

\section{DISCUSSION}

In addition to the observation by Gray (1955), mentioned in the Introduction, there have been a few other relevant observations. Tunicate spermatozoa have been observed to swim slowly $(f \sim \mathrm{I} \mathrm{Hz})$ with normal bending wave patterns after loss of the 'lateral body' containing mitochondria (Ursprung \& Schabtach, 1965; Brokaw, 1966). 
Measurements have been made of the wavelength of Ciona spermatozoa inhibited by thiourea (Brokaw \& Benedict, $1968 b$ ) and the results are included in Text-fig. 3 Although the mechanism of action of thiourea is poorly understood and the data do not cover a wide range of frequencies, the thiourea-inhibition data are consistent with the results of the present experiments. Perhaps the most significant observation of this type has been made by Gibbons \& Gibbons (1973). They found that after $\mathrm{KCl}$ extraction of half of the dynein arms from tritonated sea-urchin sperm flagella, the movements reactivated by ATP were reduced in frequency by about one half but the wavelength and amplitude of the movement were not significantly altered. Although this observation does not span a large range of frequencies, it is unique in being related to a clearly defined alteration in the internal properties of the flagellum.

We have now obtained quantitative data extending over a wider range of frequencies. In our ATP-reactivation experiments the important variable is ATP concentration. ATP concentration is most probably the important variable in the sperm-decapitation experiments and possibly also in the Triton-inhibition experiments. However, in the sperm-decapitation experiments the decrease in ATP concentration as the ATP initially in the flagellum is used up is accompanied by an increase in ADP concentration so that the total ATP + ADP concentration will not change as sharply as in the ATP-reactivation experiments. The possibility that ADP concentration effects could explain the difference between the results in our ATPreactivation experiments and our sperm-decapitation experiments could be examined further by experiments with ATP-reactivated spermatozoa in the presence of varying concentrations of $\mathrm{ADP}$. In the Triton-inhibition experiments concentrations of ADP, magnesium ion, or other internal components might change as well as the internal ATP concentration, making the interpretation of these experiments less clear.

The most general conclusion from our experiments and earlier observations is that flagella possess a mechanism which maintains the normal size and shape of the bending waves when the frequency varies. If this behaviour is an intrinsic property of the mechanism for generating flagellar bending waves, it may help us to better understand that mechanism. However, it is also possible that the constancy of wave size and shape reflects more complicated adaptations which have evolved to maintain optimum wave parameters for sperm propulsion (Brokaw, 1963).

Reduction in frequency to approximately one quarter the normal value was not accompanied by any significant change in wavelength or bend angle in the experiments with Triton-inhibited Ciona spermatozoa and decapitated Lytechinus spermatozoa. On the other hand the ATP-reactivated Lytechinus spermatozoa show a statistically significant variation in wavelength and bend angle with ATP concentration. Reactivation with about $0.2 \mathrm{mM} \mathrm{ATP}$, giving frequencies of about $6 \mathrm{~Hz}$, results in the reactivated spermatozoa having values of wavelength and bend angle which are close to the values for normal spermatozoa beating at $30 \mathrm{~Hz}$. As the ATP concentration is increased up to $3 \mathrm{mM}$, the wavelength and mean bend angle fall to about $85 \%$ and $70 \%$, respectively, of the values for normal spermatozoa.

Most Triton-inhibited Ciona spermatozoa swimming at frequencies below about one quarter the normal frequency show a small increase in wavelength and a small decrease in average curvature within bent regions so that the bend angle of the flagellar waves remains constant. This effect and the ATP concentration effect observed 
with reactivated spermatozoa represent a relatively minor, quantitative disturbance of the mechanisms which regulate wave parameters.

More extreme behaviour at low frequencies is exhibited by some of the Tritoninhibited spermatozoa, such as those illustrated in Plate I, figs. 5, 6. In these cases the wave parameters vary greatly along the length of the flagellum and between individual spermatozoa; furthermore, there is a substantial increase in wavelength although this quantity is no longer well defined. These changes suggest a more complete breakdown of the normal mechanism for regulation of wave parameters. The sharp increase in the wavelength of the decapitated Lytechinus spermatozoa at frequencies below about $3 \mathrm{~Hz}$ also suggests a relatively severe breakdown of the regulatory mechanisms. In this case also there is a decrease in average curvature within the bent regions of the bending wave since the increase in wavelength is greater than the increase in bend angle.

If flagella contain a relatively large internal viscous bending resistance, the wavelength of flagellar bending waves may be determined by the relative values of internal and external viscous resistances and will be independent of properties of the active sliding process (Brokaw, $1972 b, c, d$ ). This hypothesis could explain the constancy of wavelength over the frequency range from normal to one quarter normal frequencies, but an explanation of the increases in wavelength observed at lower frequencies would require us to assume that the internal viscous bending resistance of the flagellum becomes greater at low ATP concentrations. ATP does have a 'plasticizing' effect on muscle, and the original observations with reactivated flagella relating ATP concentration to frequency (Hoffman-Berling, I955) were interpreted as a plasticizing effect. However, with a sliding filament mechanism for flagella analogous to the sliding filament mechanism for muscle the plasticizing action of ATP should act on the crossbridges between filaments and might alter the internal viscous resistance to sliding between the filaments. Stabilization of the wavelength by internal viscous resistances is dependent on an internal viscous bending resistance rather than an internal visccus shear resistance (Brokaw, I972c). This bending resistance would appear to depend on properties of the flagellum in parallel with the active sliding process, such as the intrinsic bending resistance of the microtubular filaments in the flagellum, and there is no a priori reason to expect that these properties would vary with the ATP concentration.

The bend angles associated with the bent regions of the flagellar bending waves are a convenient measure of the amplitude of the movement and are directly related to the amount of active sliding which occurs between filaments within the flagellum. If the bend angles remain constant, the 'shape' of the bending wave is constant but its 'size' can change if the wavelength changes. An increase in wavelength without a change in bend angle causes a decrease in the curvature of the flagellum which measures the actual amplitude of bending at any point, while the actual wave amplitude, in the usual sense, will increase. Over the range of frequencies where the wavelength remains constant, all three measures of 'amplitude' of the movement remain constant but the mechanism which maintains a constant amplitude is obscure.

Gray (I955), and several more recent authors, have suggested that the elasticity of the flagellar structure may be important in determining the parameters of the bending waves propagated by flagella. In a computer simulation of a sliding-filament model for flagellar movement (Brokaw, 1972b), regulation of wave amplitude was 
achieved by a balance between active bending moments and bending moments resulting from internal elastic resistances of the flagellum. Stabilization of the wave movement at a particular amplitude depended on non-linearities in either the elastic resistances or the function controlling the moment generated by the active sliding process. In the terms of this model a reduction in frequency caused by reduced ATP concentration or removal of dynein arms from the flagellum would be interpreted as a reduction in the moment generated by the active sliding process. This reduction in active moment would lead to a balance between active and viscous bending moments being achieved at a lower frequency, and a balance between active and elastic bending moments being achieved at a lower amplitude. According to this model, reductions in both frequency and amplitude should be observed, but experimentally we find reductions in frequency without reductions in amplitude. We see three possible ways out of this dilemma:

(I) The non-linearity of the elastic resistances is so large that the amplitude is very insensitive to small variations in active moment. This explanation appears to be ruled out by the observation of significant increases in bend angle and curvature when some flagella are swimming at high viscosities (Brokaw, I966).

(2) Rather than being inert structures in parallel with the active sliding process, the elastic resistances responsible for amplitude regulation are an intrinsic property of the interfilament interaction which generates active sliding. In this case the elasticity might be decreased in proportion to the active moment by conditions which inhibit the frequency.

(3) The mechanism for amplitude regulation is independent of elastic resistances in the flagellum.

Further work is required to elucidate the mechanism of amplitude regulation in flagella.

\section{SUMMARY}

I. Treatment of Ciona spermatozoa with low concentrations of Triton X-Ioo (less than $0.01 \%$ ) causes them to beat at lower than normal frequencies. The wavelength of the flagellar bending waves remains constant over the range from 10 to $4 \circ \mathrm{Hz}$. There is a small increase in wavelength at lower frequencies; in the range of $I^{\cdot} 5-6 \cdot 2 \mathrm{~Hz}$, the wavelength averaged $114 \%$ of the normal value for Ciona spermatozoa. The angle of bend of the bent regions of the flagellar bending waves remained constant within $\pm 10 \%$ over this range of frequencies.

2. Decapitated sperm flagella from Lytechinus beat at a continually declining frequency as they exhaust their content of ATP. Both wavelength and bend angle retain normal values until the frequency falls below about $8 \mathrm{~Hz}$. Both parameters increase at lower frequencies, with a sharp increase below $3 \mathrm{~Hz}$.

3. ATP-reactivated spermatozoa from Lytechinus show relatively small changes in wavelength and bend angle as the frequency is varied over the range from 5 to $25 \mathrm{~Hz}$ by varying the $A T P$ concentration.

4. Constancy of wavelength over a wide range of frequencies is consistent with the hypothesis that wavelength is determined by the relative values of viscous bending resistance within a flagellum and external viscosity.

5. No satisfactory explanation is available at present for the constancy of bend angle 
over a wide range of frequencies nor for the changes in wave parameters which are observed at low frequencies.

This work was supported in part by a grant from the United States Public Health Service (GM 187II). We thank Lynette Bobrow for excellent assistance in the laboratory and Drs Barbara and Ian Gibbons for allowing us to read before publication their manuscript on movement of spermatozoa after partial dynein extraction.

\section{REFERENCES}

Brokaw, C. J. (1963). Movement of the flagella of Polytoma uvella. F. exp. Biol. 40, 149-56.

Brokaw, C. J. (1965). Non-sinusoidal bending waves of sperm flagella. $\mathcal{Y}$. exp. Biol. 43, $155-69$.

BROKAw, C. J. (1966). Effects of increased viscosity on the movements of some invertebrate spermatozoa. F. exp. Biol. 45, I I3-39.

BrokAw, C. J. (1967). Adenosine triphosphate usage by flagella. Science, N.Y. 156, 76-8.

BrokaN, C. J., (1970). Bending moments in free-swimming flagella. $\mathcal{F}$. exp. Biol. 53, $445-64$.

Brokaw, C. J. (197I). Bend propagation by a sliding filament model for flagella. $\mathcal{F}$. exp. Biol. 55, $289-304$.

Brokaw, C. J. (1972a). Flagellar movement: a sliding filament model. Science, N.Y. 178, 455-62.

Brokaw, C. J. $(1972 b)$. Computer simulation of flagellar movement. I. Demonstration of stable bend propagation and bend initiation by the sliding filament model. Biophys. $\mathcal{F}$. 12, 564-86.

BRokAw, C. J. (1972 c). Viscous resistance in flagella: analysis of small amplitude motion. $\mathcal{F}$. Mechanochem. Cell Mot. I, I $5 \mathrm{I}-5$.

Brokaw, C. J. $(1972 d)$. Computer simulation of flagellar movement. II. Influence of external viscosity on movement of the sliding filament model. $\mathcal{F}$. Mechanochem. Cell Mot. r, 203-I I.

Brokaw, C. J. \& Benedict, B. (I968a). Mechanochemical coupling in flagella. I. Movement-dependent dephosphorylation of ATP by glycerinated spermatozoa. Arch. biochem. Biophys. 125, 770-8.

Brokaw, C. J. \& Benedict, B. (1968 b). Mechanochemical coupling in flagella. II. Effects of viscosity and thiourea on metabolism and motility of Ciona spermatozoa. F. gen. Physiol. 52, 283-99.

Grbbons, B. H. \& GibboNs, I. R. (1972). Flagellar movement and adenosine triphosphatase activity in sea urchin sperm extracted with Triton X-10o. F. Cell Biol. 54, 75-97.

Gibbons, B. H. \& Gibbons, I. R. (I973). Effect of partial extraction of dynein arms on the movement of reactivated sea urchin sperm. $\mathscr{F}$. Cell Sci. (in press).

Gray, J. (1955). The movement of sea-urchin spermatozoa. F. exp. Biol. 32, 775-801.

Hoffman-Berling, H. (1955). Geisselmodelle und Adenosintriphosphat (ATP). Biochim. biophys. Acta I6, I 46-54.

Holwill, M. E. J. (1969). Kinetic studies of the flagellar movement of sea-urchin spermatozoa. $\mathcal{J} . \exp$. Biol. 50, 203-22.

SATIR, R. (1968). Studies on cilia. III. Further studies on the cilium tip and a sliding filament model of ciliary motility. F. Cell Biol. 39, 77-94.

Summers, K. E. \& Gibbons, I. R. (197I). Adenosine triphosphate-induced sliding of tubules in trypsintreated flagella of sea-urchin sperm. Proc. Natn. Acad. Sci. U.S.A. 68, 3092-6.

URSPRUNG, H. \& SchabTach, E. (1965). Fertilization in tunicates: loss of the parental mitochondrion prior to sperm entry. $\mathcal{F}$. exp. Zool. 159, 379-83. 


\section{EXPLANATION OF PLATE}

Figs. 1, 2. Normal movements of Ciona spermatozoa in the absence of Triton.

Fig. I: Frequency, $39 \mathrm{~Hz}$; flash frequency, $39 \cdot 7 \mathrm{~Hz}$.

Fig. 2: Frequency, $34 \mathrm{~Hz}$; flash frequency, $33 \cdot 6 \mathrm{~Hz}$.

Figs. 3, 4. Movements typical of Triton-inhibited Ciona spermatozoa.

Fig. 3: Frequency, $5.5 \mathrm{~Hz}$; flash frequency, $3.9 \mathrm{~Hz}$.

Fig. 4: Frequency, $2 \cdot 2 \mathrm{~Hz}$; flash frequency, $2 \cdot 7 \mathrm{~Hz}$.

Figs. 5, 6. Atypical movements of Triton-inhibited Ciona spermatozoa.

Fig. 5: Frequency, $2 \cdot 7 \mathrm{~Hz}$; flash frequency, $3 \cdot \circ \mathrm{Hz}$.

Fig. 6: Frequency, $3.3 \mathrm{~Hz}$; flash frequency, $3.7 \mathrm{~Hz}$.

Figs. 7-10. Movements of ATP-reactivated Lytechinus spermatozoa.

Fig. 7: $3 \mathrm{~mm} \mathrm{ATP}$. Frequency, $22 \mathrm{~Hz}$; flash frequency, $50 \mathrm{~Hz}$.

Fig. 8: I mM ATP. Frequency $18.6 \mathrm{~Hz}$; flash frequency $50 \mathrm{~Hz}$.

Fig. 9: $0.33 \mathrm{~mm}$ ATP. Frequency, $9 \mathrm{~Hz}$; flash frequency, $25_{i} \mathrm{~Hz}$.

Fig. 10: $0.11 \mathrm{mM}$ ATP. Frequency, $5.5 \mathrm{~Hz}$; flash frequency, $10 \mathrm{~Hz}$.

Figs. I I-20. Movements of the flagellum of a decapitated Lytechinus spermatozoon, taken at 2 sec intervals.

Fig. 11: Frequency, $14.5 \mathrm{~Hz}$; flash frequency, $14 \cdot 6 \mathrm{~Hz}$.

Fig. 12: Frequency, $10.6 \mathrm{~Hz}$; flash frequency, $14.6 \mathrm{~Hz}$.

Fig. 13: Frequency, 8.9 Hz; flash frequency, i1.6 Hz.

Fig. 14: Frequency, 6.1 Hz; flash frequency, $10 \cdot 4 \mathrm{~Hz}$.

Fig. 15: Frequency, $5.0 \mathrm{~Hz}$; flash frequency, $10.5 \mathrm{~Hz}$.

Fig. 16: Frequency, 3.9 Hz; flash frequency, $7 \cdot 5 \mathrm{~Hz}$.

Fig. 17: Frequency, 2.9 Hz; flash frequency, $7 \cdot 5 \mathrm{~Hz}$.

Fig. 18: Frequency, $2 \cdot 0 \mathrm{~Hz}$; flash frequency, $7 \cdot 5 \mathrm{~Hz}$.

Fig. 19; Frequency, $1 \cdot 8 \mathrm{~Hz}$; flash frequency, $7 \cdot 5 \mathrm{~Hz}$.

Fig. 20: Frequency, $1 \cdot 3 \mathrm{~Hz}$; flash frequency, $7 \cdot 5 \mathrm{~Hz}$. 
Fournal of Experimental Biology, lol. 59, No. 3
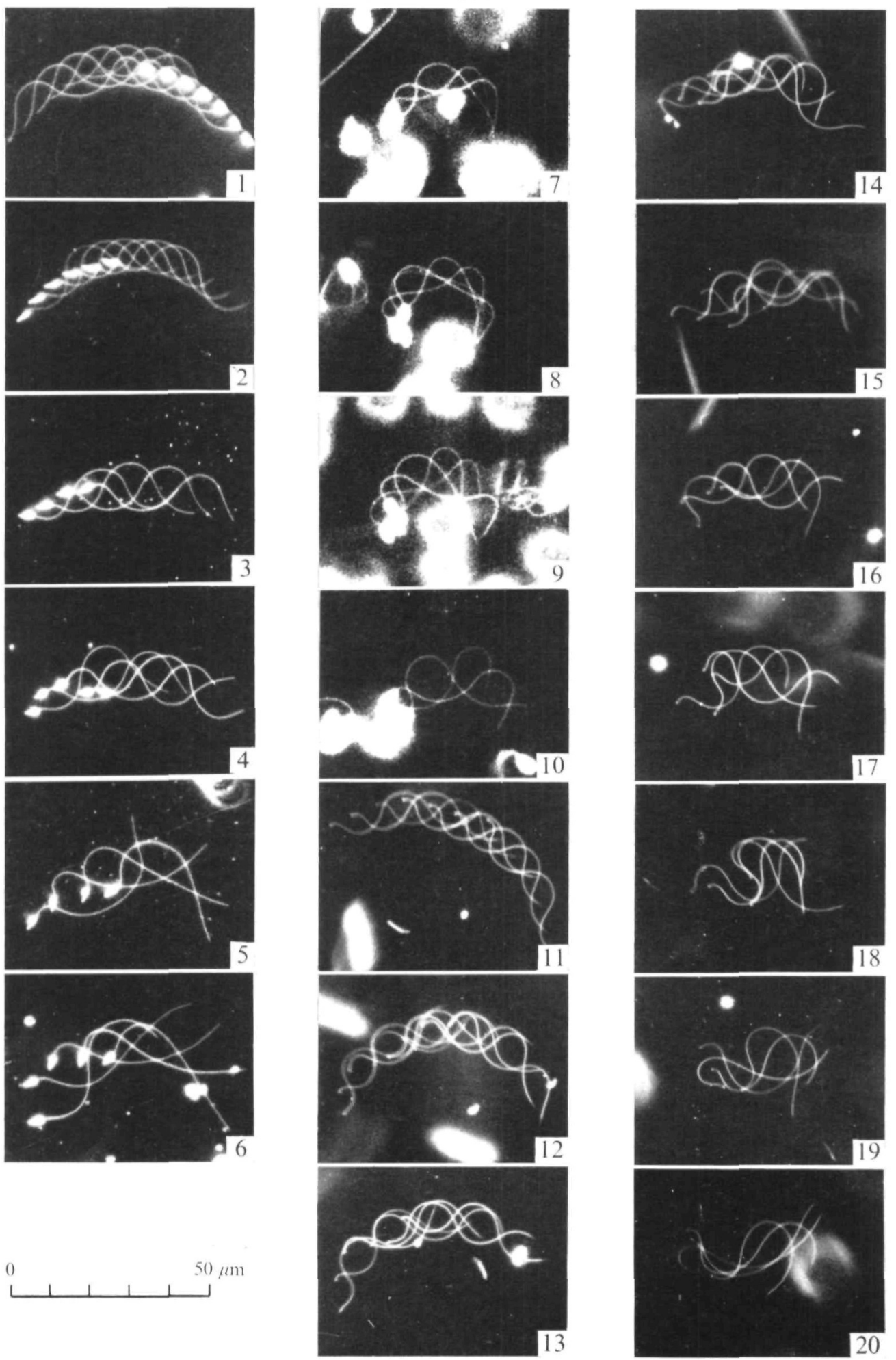

$50 \mu \mathrm{m}$
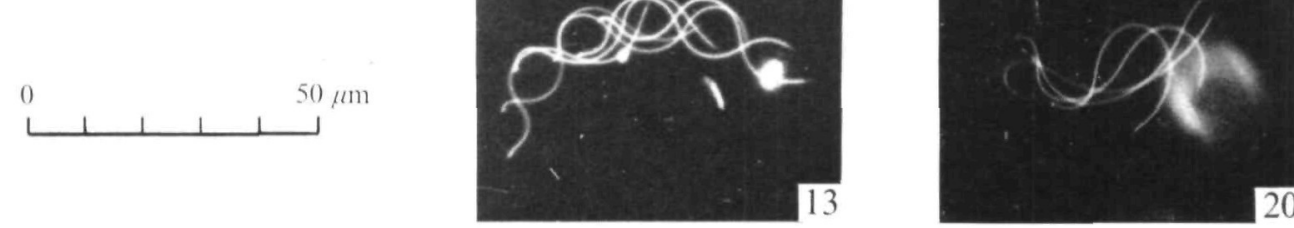

C. J. BROKAW ANU R. JOSSLIN

(Fucing P. 628) 
